\title{
Orbital Migration and the Brown Dwarf Desert
}

\author{
Philip J. Armitage \\ JILA, 440 UCB, University of Colorado, Boulder CO80309-0440, USA
}

Ian A. Bonnell

School of Physics and Astronomy, University of St Andrews, North Haugh, Fife KY16 9SS, UK

\begin{abstract}
The orbital elements of extreme mass ratio binaries will be modified by interactions with surrounding circumstellar disks. For brown dwarf companions to Solar-type stars the resulting orbital migration is sufficient to drive short period systems to merger, creating a brown dwarf desert at small separations. We highlight the similarities and the differences between the migration of brown dwarfs and massive extrasolar planets, and discuss how observations can test a migration model for the brown dwarf desert.
\end{abstract}

\section{Introduction}

Radial velocity surveys show that brown dwarfs are rarely found as close (within a few AU) binary companions to Solar-type stars. This article makes the case that the existence of this brown dwarf desert may have nothing to do with the formation of brown dwarfs. We argue instead that a brown dwarf desert is an expected consequence of the orbital migration of brown dwarfs within an evolving protostellar disk (Armitage \& Bonnell 2002). We make the basic assumption that brown dwarfs form in the same manner as stars - during gravitational collapse - and show that migration subsequently creates a desert by driving initially close star - brown dwarf binaries into mergers.

\section{Why migration matters for brown dwarfs}

A viscous accretion disk around a single star evolves such that the outward drift of a vanishingly small fraction of the mass absorbs all of the angular momentum, allowing the bulk of the disk to be accreted (e.g. Pringle 1981). The same reasoning applies to circumbinary disks (Pringle 1991). Given enough time, an arbitrarily small mass of gas, trapped outside the binary orbit, will soak up the binary orbital angular momentum, and drive the binary toward merger.

Protoplanetary disks, however, have limited lifetimes of around 1-10 Myr (Strom et al. 1989; Haisch, Lada \& Lada 2001). For rapid migration to occur, we require (roughly speaking) that the mass of gas in the disk be at least 


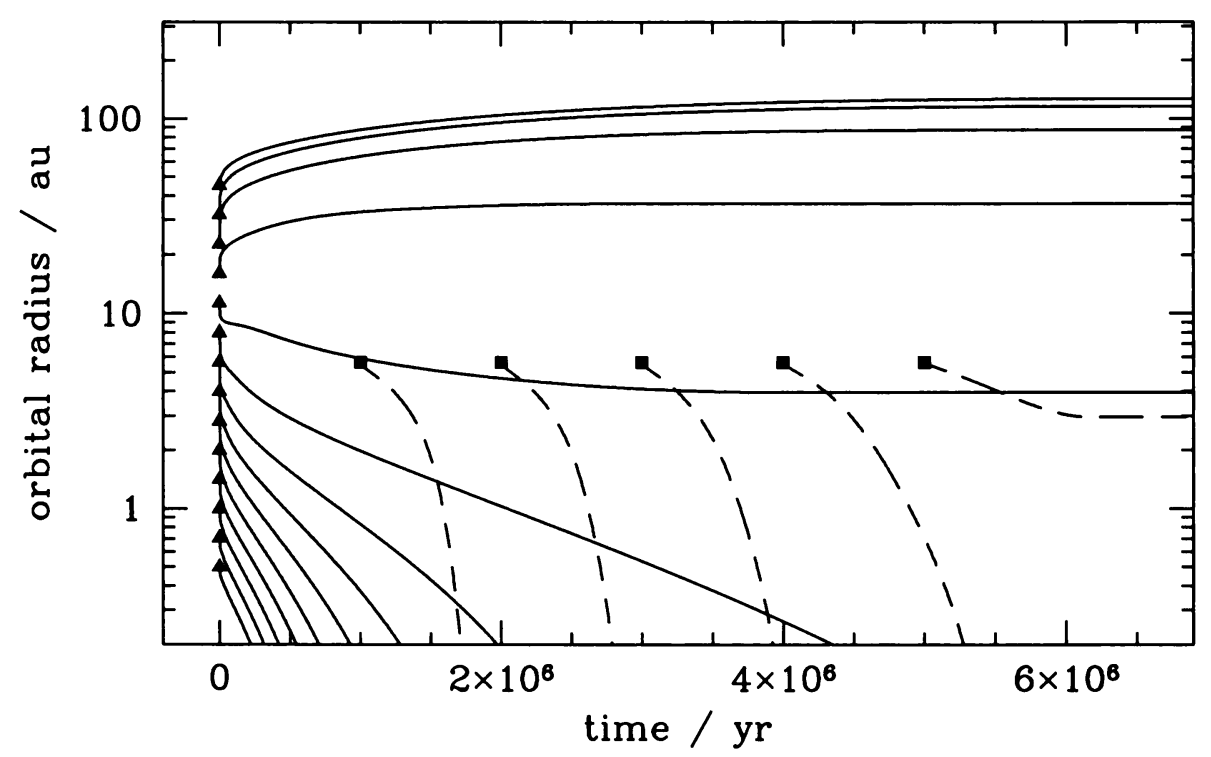

Figure 1. Comparison of the orbital evolution of $0.04 M_{\odot}$ brown dwarfs (solid lines) and Jupiter mass planets (dashed lines) embedded within an evolving protoplanetary disk. Brown dwarfs form early, so to survive migration they must form within a narrow range of disk radii. Their evolutionary tracks diverge, implying that migration reduces the number of brown dwarfs at small orbital radii - forming a brown dwarf desert. Planets are even more susceptible to migration, but they can survive to be observed at radii of a few AU by forming at a later epoch, when the disk is weaker and unable to drive significant migration.

comparable to the mass of the embedded substellar object ${ }^{1}$. This requirement is almost certainly met for brown dwarfs that are binary companions to Solartype stars, since both observations (Osterloh \& Beckwith 1995) and theoretical arguments (Lin \& Pringle 1990) suggest that the disk mass at early times comfortably exceeds typical brown dwarf masses. We therefore expect migration to be significant for brown dwarfs orbiting Solar mass stars, though not for brown dwarfs embedded in the weaker disks surrounding very low mass stars.

\section{Forming a brown dwarf desert}

To make quantitative predictions for the impact of migration on brown dwarfs, we have used a one dimensional model that was developed to study planetary migration (Armitage et al. 2002; see also Trilling et al. 1998; Trilling, Lunine

\footnotetext{
${ }^{1}$ This condition can be easily understood. If the angular momentum of the substellar object greatly exceeds that of the disk, it will take many viscous times for the disk to transport the 'extra' angular momentum to large radii, and migration will be very slow.
} 
\& Benz 2002). We solve for the coupled evolution of a viscous protoplanetary disk (using a variant of the disk model of Clarke, Gendrin \& Sotomayor 2001) and an embedded brown dwarf. We assume that the brown dwarf orbit remains circular, not because we believe this to be true (Papaloizou, Nelson \& Masset 2001; Goldreich \& Sari 2002), but because of the difficulty of doing better. We also assume that the brown dwarf does not accrete further mass from the disk during migration. For circular orbits, this is likely to be a reasonable approximation for substellar objects with masses above a few Jupiter masses (Lubow, Seibert \& Artymowicz 1999).

Figure 1 shows the extent of the resulting migration for $0.04 M_{\odot}$ brown dwarfs embedded in disks whose initial mass is $0.1 M_{\odot}$. Brown dwarfs with initial orbital radii smaller than about $5 \mathrm{AU}$ are all swept in to small radii and probably merge with the star (conceivably, as has been suggested for extrasolar planets, some survive at very small radii as the brown dwarf equivalent of hot Jupiters). At larger radii, where the disk is expanding, outward migration occurs and pushes brown dwarfs out to radii of $\sim 10^{2}$ AU. Between these extremes, brown dwarfs formed within a small window of initial orbital radii spread out to populate all short period orbits. This dilution of $\mathrm{d} N / \mathrm{d} \log a$ at small radii leads to a brown dwarf desert within this model.

We do not expect the desert - if it results from migration - to be completely devoid of brown dwarfs. A handful of brown dwarfs should end up in short period orbits having migrated inward from initially larger disk radii. Depending upon the assumed initial (pre-migration) distribution of brown dwarf orbital radii, we have estimated that the frequency of brown dwarfs within 4 AU should be depleted by a factor of 5-10 (Armitage \& Bonnell 2002).

Why is there a brown dwarf desert at the same radii where there is an abundance of massive extrasolar planets (Marcy \& Butler 2000)? The arguments given above suggest - correctly - that the lower masses of extrasolar planets should make them even more vulnerable to migration than brown dwarfs. Massive planets, however, probably do not form via the same mechanism as brown dwarfs (though for contrary opinions see Boss 1998; Armitage \& Hansen 1999). Conventionally, they form instead via core accretion, a slow process that may take several Myr, especially in the outer parts of the disk. As shown in Figure 1, this allows planets to escape destruction via mergers by forming at a later epoch, when the protoplanetary disk is of lower mass and unable to force the planets into mergers.

\section{Observational tests}

We have argued that one should not conclude, based solely on the existence of the brown dwarf desert, that brown dwarfs cannot form as close binary companions to Solar-type stars. Migration at a later epoch can accomplish the same end result, and makes several additional predictions.

(i) Migration occurs if the binary mass ratio $q=M_{\mathrm{BD}} / M_{*}$ is comparable to or less than the disk mass ratio $M_{\text {disk }} / M_{*}$. This is unlikely to be true for brown dwarfs around very low mass stars. Therefore, no brown dwarf desert is expected around M stars. 
(ii) The same argument applies to $M$ star companions to stars of a few Solar masses - if the latter have disks that live for several viscous times. Migration could clear out a low mass stellar desert at small separations around stars modestly more massive than the Sun.

(iii) Migration takes of the order of a Myr. No brown dwarf desert is expected around the youngest pre-main-sequence stars.

We emphasize that the first two predictions are not unique to this model. Accretion onto forming binary systems also abhors extreme mass ratios (Bate 2000), and provides an alternative theoretical explanation for the brown dwarf desert. The third prediction is more discriminatory. Detection of a higher frequency of brown dwarfs around young pre-main-sequence stars would provide strong support for the model presented here. Competing theories based on differential accretion (Bate 2000) and dynamical interactions (Reipurth \& Clarke 2001) predict that the desert is established on a dynamical timescale, which is at least an order of magnitude shorter than the timescale on which migration occurs.

\section{References}

Armitage, P. J., \& Bonnell, I. A. 2002, MNRAS, 330, 11

Armitage, P. J., \& Hansen, B. M. S. 1999, Nature, 402, 633

Armitage, P. J., Livio, M., Lubow, S. H., \& Pringle, J. E. 2002, MNRAS, 334, 248

Bate, M. R. 2000, MNRAS, 314, 33

Boss, A. P. 1998, ApJ, 503, 923

Clarke, C. J., Gendrin, A., \& Sotomayor, M. 2001, MNRAS, 328, 485

Goldreich, P., \& Sari, R. 2002, ApJ, submitted

Haisch, K. E., Lada, E. A., \& Lada, C. J. 2001, ApJ, 553, L153

Lin, D. N. C., \& Pringle, J. E. 1990, ApJ, 358, 515

Lubow, S. H., Seibert, M., \& Artymowicz, P. 1999, ApJ, 526, 1001

Marcy, G. W., \& Butler, R. P. 2000, PASP, 112, 137

Osterloh, M., \& Beckwith, S. V. W. 1995, ApJ, 439, 288

Papaloizou, J. C. B., Nelson, R. P., \& Masset, F. 2001, A\&A, 366, 263

Pringle, J. E. 1981, ARA\&A, 19, 137

Pringle, J. E. 1991, MNRAS, 248, 754

Reipurth, B., \& Clarke, C. J. 2001, AJ, 122, 432

Strom, K. M., Strom, S. E., Edwards, S., Cabrit, S., \& Skrutskie, M. F. 1989, AJ, 97, 1451

Trilling, D. E., Benz, W., Guillot, T., Lunine, J. I., Hubbard, W. B., \& Burrows, A. 1998, ApJ, 500, 428

Trilling, D. E., Lunine, J. I., \& Benz, W. 2002, A\&A, submitted 